\title{
Dynamics of stochastic Lorenz family of chaotic systems with jump
}

\author{
Zaitang Huang · Junfei Cao • Ting Jiang
}

Received: 13 September 2013 / Accepted: 8 November 2013 / Published online: 24 November 2013 C) The Author(s) 2013. This article is published with open access at Springerlink.com

\begin{abstract}
This paper discusses the Lorenz family of chaotic systems under the influence of poisson noise. We obtain the sufficient and necessary conditions for stochastic stability under some assumptions for stochastic Lorenz family of chaotic systems with jump. We then investigate the estimation of the global attractive set and stochastic bifurcation behavior of the family of stochastic Lorenz system. Numerical experiments illustrate the results.
\end{abstract}

Keywords Random attractor - Stochastic bifurcation - Stochastic stability . Stochastic Lorenz systems · Poisson noise

\section{Introduction}

The Lorenz attractor (also known as butterfly attractor) was found in 1963 by E. N. Lorenz [1] for a dynamical system generated by a three dimensional autonomous differential equation describing a meteorological problem, he has created a new era of nonlinear dynamical systems [2-10]. A classical Lorenz family of chaotic systems has the following form:

$$
\left\{\begin{array}{l}
\dot{x}(t)=a(y-x) \\
\dot{y}(t)=c x-x z-d y \\
\dot{z}(t)=x y-b z
\end{array}\right.
$$

\section{Z. Huang $(\bowtie) \cdot$ T. Jiang}

School of Mathematical Sciences, Guangxi Teachers Education University, Nanning 530023, People's Republic of China

e-mail: zaitanghuang@163.com

J. Cao

Department of Mathematics, Guangdong University of Education,

Guangzhou 510310, People's Republic of China 
with initial condition $\left(x_{0}, y_{0}, z_{0}\right)=u_{0}$ and parameters $a, b$ and $d$ are positive constants, $c \in(-\infty,+\infty)$. To study the qualitative behavior of a Lorenz family of chaotic system, the property of attractors play an important role in the study of the asymptotic behavior of Lorenz family of chaotic systems. Many scholars have studied Lorenz family of chaotic systems carefully. Tucker [11] firstly rigorously proved existence of the Lorenz attractor, he pointed out that it was very difficult to obtain information of the attractor directly from the differential equation itself. The Russian scholar, Leonov $[12,13]$ was the first one to investigate the estimation of the global attractive set of the Lorenz system. Liao et al. $[14,15]$ discussed the stability and global attractive set of the Lorenz system. Li et al. [16] applied the method of Lagrange multiplier to obtain an estimation of the global attractive set for the Lorenz system. For stochastic chaotic systems, however, comparably little progress has been made by now.

An important chaotic systems component in nature is that chaotic systems are inevitably affected by environmental noises. Arnold, [17] has pointed out that the parameters in the chaotic systems exhibit random fluctuation to a greater or lesser extent due to various environmental noises. Currently, one of the important ways to model the in influence of the environmental fluctuations in chaotic systems is to assume that the random noises affect the parameters. Scholars usually estimate them by average values plus some error terms. In general, by the well-known central limit theorem, the error terms follow normal distributions. That is

$$
\begin{aligned}
& a x \rightarrow a x-\sigma_{1} x d W(t), \quad a y \rightarrow a y+\gamma_{1} x d N(t), \\
& c x \rightarrow c x+\gamma_{2} x d N(t), \quad d y \rightarrow d y-\sigma_{2} y d W(t),
\end{aligned}
$$

where $W(t)$ are mutually independent Brownian motions, $N(t)$ is a Poisson process with intensity $\lambda, \sigma_{1}, \sigma_{2}, \gamma_{1}$ and $\gamma_{2}$ represent the intensities of random noises. Then, the corresponding Itô's-type family of the stochastic Lorenz system with jumps becomes:

$$
\left\{\begin{array}{l}
d x(t)=[a(y-x)] d t+\sigma_{1} x d W(t)+\gamma_{1} y d N(t) \\
d y(t)=[c x-x z-d y] d t+\sigma_{2} y d W(t)+\gamma_{2} x d N(t) \\
d z(t)=[x y-b z] d t
\end{array}\right.
$$

As the stochastic disturbances are unavoidable, it is reasonable to study the stochastic stability and stochastic bifurcations for the family of stochastic Lorenz system (1.2). Keller [18] have studied attractors and bifurcation of the stochastic Lorenz system. Schmalfuß estimate the Hausdorff dimension of the attractor [19]. Global stability of the family of Lorenz system has been studied by a lot of authors [20,21]. Most of the papers consider the situations at which deterministic Lorenz system are present. Essentially, the point is globally asymptotically stable if there is the domination of the deterministic Lorenz system. However, we find few papers referring to how the sharp domination is. In other words, there are few studies giving necessary and sufficient conditions for the global stability of stochastic Lorenz family of chaotic systems. In this paper, we obtain the sufficient and necessary conditions for stochastic stability under some assumptions for stochastic Lorenz family of chaotic systems with jump. As theory of stochastic bifurcation is still in its infancy [22,23], there are few rigorous general theorems, and many phenomena have been only by computer 
simulations, or for particular models. In particular, some bifurcation phenomena were observed numerically [24], experimentally, and by approximations based on averaging, phenomenological or scaling methods [25-29]. In this paper, we investigate the estimation of the global attractive set by Lyapunov direct method, and we show that random attractors provide an important tool to analyze the bifurcation behavior of the stochastically perturbed family of Lorenz system [30,31].

\section{Preliminaries and notations}

Let $\{\Omega, \mathcal{F}, \mathbb{P}\}$ be are probability space. On $\Omega$ we define a flow $\theta$ of maps $\theta_{t}: \Omega \rightarrow \Omega$ with $t \in R$, i.e.

$$
\theta_{0}=i d_{\Omega} \quad \theta_{t} \circ \theta_{s}=\theta_{t+s} \quad s, t \in R,
$$

(for brevity we write $\left.\theta_{t} \circ \theta_{s}=\theta_{t} \theta_{s}\right)$ such that $(t, \omega) \rightarrow \theta_{t} \omega$ is $\mathcal{F} \otimes \mathcal{B}(R)$-measurable and $\theta_{t} \mathbb{P}=\mathbb{P}$ (measure preserving). In addition $\mathbb{P}$ is assumed to be ergodic w.r.t. the flow $\theta$. We call $\left\{\Omega, \mathcal{F}, \mathbb{P}, \theta_{t \in R}\right\}$ or $\theta$ for short, a metric dynamical system.

Definition 2.1 A function $\phi: T \times \Omega \times R^{d} \rightarrow R^{d}$ is called a random dynamical $\operatorname{system}(R D S)$ over the metric dynamical system $\theta$, if $\phi$ is $\mathcal{B}(\mathbb{T}) \otimes \mathcal{F} \otimes \mathcal{B}\left(\mathbb{R}^{\ltimes}\right)$, $\mathcal{B}\left(\mathbb{R}^{\ltimes}\right)$-measurable and if the mappings $\phi(t, \omega):=\phi(t, \omega, \cdot): R^{d} \rightarrow R^{d}$ form a cocycle over $\theta$, i.e. for all $\omega \in \Omega$

$$
\theta(0, \omega)=i d_{R^{d}} \quad \phi(t+s, \omega)=\phi\left(t, \theta_{s} \omega\right) \circ \phi(s, \omega), \quad s, t \in \mathbb{T} .
$$

Further, $\phi$ is called continuous $R D S$, if $\phi(t, \omega)$ is continuous for all $\omega \in \Omega, t \in \mathbb{T}$.

Definition 2.2 A random variable $x: \Omega \rightarrow R^{d}$ is called a random fixed point (or stationary solution) of a given $R D S \phi$ if $\phi(t, \omega) x(\omega)=x\left(\theta_{t} \omega\right)$, for all $\omega \in \Omega, t \in \mathbb{T}$.

Definition 2.3 A random compact set $D(\omega)$ maps $\Omega$ into the space of nonempty compact subset of $\mathbb{R}^{n}$ such that for all $x \in \mathbb{R}^{n}$ the mapping $\omega \rightarrow d(x, D(\omega))$ is measurable, where $d(x, D(\omega))=\inf _{y \in D(\omega)}\|x-y\|$.

A nonempty family $\mathcal{D}$ consisting of the random compact sets is called inclusion closed system (IC-system), if it is maximal w.r.t. to inclusion (i.e. if $D \in \mathcal{D}$ and $D^{\prime}(\omega) \subset D(\omega)$ for all $\omega \in \Omega$ is a random compact set then also $\left.D^{\prime} \in \mathcal{D}\right)$.

Definition 2.4 Let $\mathcal{D}$ be an IC-system. A random compact set $B \in \mathcal{D}$ is called $\mathcal{D}$-absorbing for an $R D S \phi$, if for only $\omega \in \Omega$ and $D \in \mathcal{D}$ there exist a $\tau_{D}(\omega)$ such that $\phi\left(t, \theta_{-t} \omega, D\left(\theta_{-t} \omega\right)\right) \subset B(\omega)$, for all $t>\tau_{D}(\omega)$. where $\phi(t, \omega) A:=$ $\bigcup_{y \in A} \phi(t, \omega, x)$. for all $A \subset R^{n}$.

Similarly we now can define a random attractor.

Definition 2.5 Let $\mathcal{D}$ be an IC-system. A random compact set $A \in \mathcal{D}$ is called $\mathcal{D}$-attractor of an $R D S \phi$, if

(i) $A$ is invariant, i.e. $\phi(t, \omega, A(\omega))=A\left(\theta_{t} \omega\right)$, for allt $>0, \omega \in \Omega$. 
(ii) $A$ is $\mathcal{D}$-attracting, i.e. for all $\omega \in \Omega$ and $D \in \mathcal{D}$

$$
\lim _{t \rightarrow+\infty} \operatorname{dist}\left(\phi\left(t, \theta_{-t} \omega, D\left(\theta_{-t} \omega\right)\right), B(\omega)\right)=0
$$

where $\operatorname{dist}(A, B)=\sup _{x \in A} \inf _{y \in B} d(x, y)$ is the usual Hausdorff semi-metric.

Theorem 2.1 [31] Let $\phi$ be continuous $R D S$ and let $\mathcal{D}$ be an IC-system, Moreover let $B \in \mathcal{D}$ be an random compact set which is $\mathcal{D}$-absorbing. Then there exist a unique $\mathcal{D}$-attractor $A \in \mathcal{D}$ for the cocycle $\phi$ given by

$$
A(\omega)=\bigcap_{t \geq 0} \overline{\bigcup_{t \geq \tau} \phi\left(\tau, \theta_{-\tau} \omega\right), B\left(\theta_{-\tau} \omega\right)}
$$

If $B(\omega)$ is connected then so is $A(\omega)$.

\section{Stochastic stability}

The purpose of this section is to seek the necessary and sufficient condition for the global asymptotically exponential stable of the equilibrium point $S_{0}(0,0,0)$ of (1.2). The result is the following:

Theorem 1 The equilibrium point $S_{0}(0,0,0)$ of $(1.2)$ is globally asymptotically exponential stable for all $a>0, b>0, d>0, \sigma_{i}>0, \gamma_{i}>0, c \in(-\infty,+\infty)$ if

$$
L=\frac{\max \{\kappa,-b\}}{\min \left\{\frac{1}{a}, \frac{1}{2}\right\}}+\lambda \log \left(1+\frac{\frac{1}{2} \max \left\{\left(\frac{1}{a} \gamma_{1}+\gamma_{2}+1\right),\left(\frac{1}{a} \gamma_{1}+\gamma_{2}+\frac{1}{a}\right)\right\}}{\min \left\{\frac{1}{a}, \frac{1}{2}\right\}}\right)<0
$$

where

$$
\kappa=\frac{\sigma_{1}^{2}-2 a+a \sigma_{2}^{2}-2 a d+\sqrt{\left(\sigma_{1}^{2}-a\left(2+\sigma_{2}^{2}-2 d\right)\right)^{2}+4 a^{2}(c+1)^{2}}}{4 a}<0
$$

Proof Define the Lyapunov function on $\Re^{3}$

$$
V(x, y, z)=\frac{1}{2}\left(\frac{1}{a} x^{2}+y^{2}+z^{2}\right) .
$$

Obviously,

$$
\begin{aligned}
& \min \left\{\frac{1}{2 a}, \frac{1}{2}\right\}\left(x^{2}+y^{2}+z^{2}\right) \leq \frac{1}{2}\left(\frac{1}{a} x^{2}+y^{2}+z^{2}\right) \\
& \leq \max \left\{\frac{1}{2 a}, \frac{1}{2}\right\}\left(x^{2}+y^{2}+z^{2}\right) .
\end{aligned}
$$


Applying Itô's formula yields, we have

$$
\begin{aligned}
& d V(x, y, z)=\left[-x^{2}-d y^{2}+(c+1) x y-b z^{2}+\frac{\sigma_{1}^{2}}{2 a} x^{2}+\frac{\sigma_{2}^{2}}{2} y^{2}\right] d t \\
& +\left(\frac{\sigma_{1}}{2 a} x^{2}+\frac{\sigma_{2}}{2} y^{2}\right) d W(t)+\frac{1}{2}\left[\frac{1}{a}\left(2 \gamma_{1} x y+y^{2}\right)+2 \gamma_{2} x y+x^{2}\right] d N_{t} \\
& \leq\left[-x^{2}-d y^{2}+(c+1) x y-b z^{2}+\frac{\sigma_{1}^{2}}{2 a} x^{2}+\frac{\sigma_{2}^{2}}{2} y^{2}\right] d t \\
& +\left(\frac{\sigma_{1}}{2 a} x^{2}+\frac{\sigma_{2}}{2} y^{2}+z^{2}\right) d W(t) \\
& +\frac{1}{2}\left[\left(\frac{1}{a} \gamma_{1}+\gamma_{2}+1\right) x^{2}+\left(\frac{1}{a} \gamma_{1}+\gamma_{2}+\frac{1}{a}\right) y^{2}+z^{2}\right] d N_{t} \\
& \leq\left[\max \{\kappa,-b\}\left(x^{2}+y^{2}+z^{2}\right)\right] d t+\max \left\{\frac{\sigma_{1}}{2 a}, \frac{\sigma_{2}}{2}, 1\right\} \\
& \times\left(x^{2}+y^{2}+z^{2}\right) d W(t) \\
& +\frac{1}{2} \max \left\{\left(\frac{1}{a} \gamma_{1}+\gamma_{2}+1\right),\left(\frac{1}{a} \gamma_{1}+\gamma_{2}+\frac{1}{a}\right), 1\right\}\left(x^{2}+y^{2}+z^{2}\right) d N_{t} \\
& \leq\left[\frac{\max \{\kappa,-b\}}{\min \left\{\frac{1}{2 a}, \frac{1}{2}\right\}} V(x, y, z)\right] d t+\frac{\max \left\{\frac{\sigma_{1}}{2 a}, \frac{\sigma_{2}}{2}, 1\right\} V(x, y, z)}{\min \left\{\frac{1}{2 a}, \frac{1}{2}\right\}} d W(t) \\
& +\frac{\frac{1}{2} \max \left\{\left(\frac{1}{a} \gamma_{1}+\gamma_{2}+1\right),\left(\frac{1}{a} \gamma_{1}+\gamma_{2}+\frac{1}{a}\right), 1\right\}}{\min \left\{\frac{1}{2 a}, \frac{1}{2}\right\}} V(x, y, z) d N_{t}
\end{aligned}
$$

where

$$
\kappa=\frac{\sigma_{1}^{2}-2 a+a \sigma_{2}^{2}-2 a d+\sqrt{\left(\sigma_{1}^{2}-a\left(2+\sigma_{2}^{2}-2 d\right)\right)^{2}+4 a^{2}(c+1)^{2}}}{4 a} .
$$

From (3.2) and Doléans-Dade's exponent formula, we can obtain

$$
V(x, y, z) \leq V\left(x_{0}, y_{0}, z_{0}\right) \exp \left\{\alpha t+\beta W(t)+\log (1+\gamma) N_{t}\right\},
$$

where

$$
\begin{aligned}
& \alpha=\frac{\max \{\kappa,-b\}}{\min \left\{\frac{1}{2 a}, \frac{1}{2}\right\}} \beta=\frac{\max \left\{\frac{\sigma_{1}}{2 a}, \frac{\sigma_{2}}{2}, 1\right\}}{\min \left\{\frac{1}{2 a}, \frac{1}{2}\right\}}, \\
& \gamma=\frac{\frac{1}{2} \max \left\{\left(\frac{1}{a} \gamma_{1}+\gamma_{2}+1\right),\left(\frac{1}{a} \gamma_{1}+\gamma_{2}+\frac{1}{a}\right), 1\right\}}{\min \left\{\frac{1}{2 a}, \frac{1}{2}\right\}} .
\end{aligned}
$$


Then we have the top Lyapunov exponent $L$

$$
\begin{aligned}
L & =\lim _{t \rightarrow \infty} \frac{\log (V(x, y, z))}{t} \leq \lim _{t \rightarrow \infty} \frac{\alpha t+\beta W(t)+\log (1+\gamma) N_{t}}{t} \\
& =\alpha+\lambda \log (1+\gamma)<0
\end{aligned}
$$

Thus, we can obtain

$$
\begin{aligned}
\min \left\{\frac{1}{2 a}, \frac{1}{2}\right\} \mathbb{E}\left(x^{2}+y^{2}+z^{2}\right) \leq & \mathbb{E} V(x, y, z) \leq V\left(x_{0}, y_{0}, z_{0}\right) \\
& \times \exp \left\{[\alpha+\lambda \log (1+\gamma)]\left(t-t_{0}\right)\right\} \\
\leq & \max \left\{\frac{1}{2 a}, \frac{1}{2}\right\}\left(x_{0}^{2}+y_{0}^{2}+z_{0}^{2}\right) \\
& \times \exp \left\{[\alpha+\lambda \log (1+\gamma)]\left(t-t_{0}\right)\right\} .
\end{aligned}
$$

Therefore,

$$
\mathbb{E}\left(x^{2}+y^{2}+z^{2}\right) \leq \frac{\max \left\{\frac{1}{2 a}, \frac{1}{2}\right\}}{\min \left\{\frac{1}{2 a}, \frac{1}{2}\right\}}\left(x_{0}^{2}+y_{0}^{2}+z_{0}^{2}\right) \exp \left\{[\alpha+\lambda \log (1+\gamma)]\left(t-t_{0}\right)\right\}
$$

Then, the equilibrium point $S_{0}(0,0,0)$ of (1.2) is globally asymptotically exponential stable. The proof of the theorem is complete.

Theorem 2 The equilibrium point $S_{0}(0,0,0)$ of (1.2) is globally asymptotically exponential stable for all $a>\sigma^{2} / 2, d=1,0 \leq \sigma_{1}=\sigma_{2}=\sigma \leq \sqrt{2}, \gamma_{i}=0, c \in$ $(-\infty,+\infty)$ if only and if

$$
c<\sqrt{\frac{\left(2 a-\sigma^{2}\right)\left(2-\sigma^{2}\right)}{a}}-1 .
$$

Proof Sufficiency. (i) Let $0 \leq c<\sqrt{\frac{\left(2 a-\sigma^{2}\right)\left(2-\sigma^{2}\right)}{a}}-1$. Define the Lyapunov function on $\mathfrak{R}^{3}$

$$
V(x, y, z)=\frac{1}{2}\left(\frac{1}{a} x^{2}+y^{2}+z^{2}\right)
$$

By Theorem 1, we have

$$
\mathbb{E}\left(x^{2}+y^{2}+z^{2}\right) \leq \frac{\max \left\{\frac{1}{2 a}, \frac{1}{2}\right\}}{\min \left\{\frac{1}{2 a}, \frac{1}{2}\right\}}\left(x_{0}^{2}+y_{0}^{2}+z_{0}^{2}\right) \exp \left[\alpha\left(t-t_{0}\right)\right],
$$


where

$$
\begin{aligned}
& \alpha=\frac{\max \{\kappa,-b\}}{\min \left\{\frac{1}{2 a}, \frac{1}{2}\right\}}, \\
& \kappa=\frac{\sigma_{1}^{2}-2 a+a \sigma_{2}^{2}-2 a d+\sqrt{\left(\sigma_{1}^{2}-a\left(2+\sigma_{2}^{2}-2 d\right)\right)^{2}+4 a^{2}(c+1)^{2}}}{4 a}<0 .
\end{aligned}
$$

(ii) $c<0$. Choose the Lyapunov function on $\mathfrak{R}^{3}$

$$
V(x, y, z)=\frac{1}{2}\left(-\frac{c}{a} x^{2}+y^{2}+z^{2}\right) .
$$

Then

$\min \left\{-\frac{c}{2 a}, \frac{1}{2}\right\}\left(x^{2}+y^{2}+z^{2}\right) \leq V(x, y, z) \leq \max \left\{-\frac{c}{2 a}, \frac{1}{2}\right\}\left(x^{2}+y^{2}+z^{2}\right)$.

Itô's formula yields

$$
\begin{aligned}
d V(x, y, z)= & {\left[\left(c-\frac{c \sigma^{2}}{2 a}\right) x^{2}-y^{2}-b z^{2}+\frac{\sigma^{2}}{2} y^{2}\right] d t+\left(-\frac{c \sigma}{2 a} x^{2}+\frac{\sigma}{2} y^{2}\right) d W(t) } \\
\leq & \max \left\{-b, \frac{\sigma^{2}}{2}-1,\left(c-\frac{c \sigma^{2}}{2 a}\right)\right\}\left(x^{2}+y^{2}+z^{2}\right) d t \\
& +\sigma\left(-\frac{c}{2 a} x^{2}+\frac{1}{2} y^{2}+z^{2}\right) d W(t) \\
\leq & \frac{\max \left\{-b, \frac{\sigma^{2}}{2}-1,\left(c-\frac{c \sigma^{2}}{2 a}\right)\right\}}{\min \left\{-\frac{c}{2 a}, \frac{1}{2}\right\}} V(x, y, z) d t+\sigma V(x, y, z) d W(t) .
\end{aligned}
$$

Thus, we have

$$
E V(x, y, z) \leq V\left(x_{0}, y_{0}, z_{0}\right) \exp \left\{\frac{\max \left\{-b, \frac{\sigma^{2}}{2}-1,\left(c-\frac{c \sigma^{2}}{2 a}\right)\right\}}{\min \left\{-\frac{c}{2 a}, \frac{1}{2}\right\}}\left(t-t_{0}\right)\right\},
$$

then

$$
\begin{aligned}
\mathbb{E}\left(x^{2}+y^{2}+z^{2}\right) \leq & \max \left\{-\frac{c}{2 a}, \frac{1}{2}\right\}\left(x_{0}^{2}+y_{0}^{2}+z_{0}^{2}\right) \\
& \times \exp \left\{\frac{\max \left\{-b, \frac{\sigma^{2}}{2}-1,\left(c-\frac{c \sigma^{2}}{2 a}\right)\right\}}{\min \left\{-\frac{c}{2 a}, \frac{1}{2}\right\}}\left(t-t_{0}\right)\right\} .
\end{aligned}
$$


Therefore, for $0 \leq c<\sqrt{\frac{\left(2 a-\sigma^{2}\right)\left(2-\sigma^{2}\right)}{a}}-1$ or $c<0$, then the equilibrium point $S_{0}(0,0,0)$ of $(1.2)$ is globally asymptotically exponential stable.

Necessity. The linearization of the Eq. (1.2) at $S_{0}(0,0,0)$ is

$$
d X=\left(\begin{array}{lll}
-a+\frac{\sigma^{2}}{2} & a & 0 \\
c & -1+\frac{\sigma^{2}}{2} & 0 \\
0 & 0 & -b
\end{array}\right) X+\sigma\left(\begin{array}{lll}
1 & 0 & 0 \\
0 & 1 & 0 \\
0 & 0 & 0
\end{array}\right) X \circ d W(t)
$$

The deterministic eigenvalues are $\lambda_{3}=-b$ and

$$
\lambda_{1,2}=-\frac{a+1}{2} \pm \frac{1}{2} \sqrt{(a+1)^{2}+4 a(c-1)} .
$$

The third equation in (3.10) is always stable and decoupled from the first two equations which, after transformation of the drift matrix to diagonal form, reads

$$
d X=\left(\begin{array}{ll}
\lambda_{3} & 0 \\
0 & \lambda_{4}
\end{array}\right) X+\sigma\left(\begin{array}{ll}
1 & 0 \\
0 & 1
\end{array}\right) X \circ d W(t)
$$

where

$$
\lambda_{3,4}=\frac{-1-a+\sigma^{2} \pm \sqrt{1-2 a+a^{2}+4 a c}}{2} .
$$

For small intensity parameter $\sigma$ Theorem 9.4.11 [22] yields for the top Lyapunov exponent of (3.11) and of (3.10)

$$
L(c, \sigma)=\frac{-1-a+\sigma^{2} \pm \sqrt{1-2 a+a^{2}+4 a c}}{2} .
$$

Since the equilibrium point $S_{0}(0,0,0)$ of (1.2) is globally asymptotically exponential stable, then the equilibrium point $S_{0}(0,0,0)$ of $(1.2)$ is local exponential stable. That is

$$
\overline{L(c, \sigma)}=\frac{-1-a+\sigma^{2}+\sqrt{1-2 a+a^{2}+4 a c}}{2}<0 .
$$

Then, we have $c<1-\sigma^{2} / 2-\sigma^{2}\left(2-\sigma^{2}\right) \Rightarrow c<\sqrt{\frac{\left(2 a-\sigma^{2}\right)\left(2-\sigma^{2}\right)}{a}}-1$. The proof of the theorem is complete.

\section{Random attractor}

In this section we will prove the existence of random attractors for the family of the stochastic Lorenz system with jumps. 
Theorem 3 Let $a>0, b>2 d, c>0, d>0, a-d>\frac{\sigma_{1}^{2}-\sigma_{2}^{2}}{2}$,

$$
L_{\mu}=\frac{b^{2}(\mu a+c)^{2}}{4 \alpha(b-d)}, \quad \alpha=\left(d-\frac{\sigma_{2}^{2}}{2}\right)-\lambda \max \left\{\gamma_{1}^{2}+2 \gamma_{1}, \gamma_{2}^{2}+2 \gamma_{2}\right\}>0
$$

then the following estimate holds on the system (1.2)

$$
\mathbb{E}\left[V_{\mu}(X)-L_{\mu}\right] \leq\left(V_{\mu}\left(X_{0}\right)-L_{\mu}\right) e^{-2 d\left(t-t_{0}\right)}
$$

In particular,

$\Omega_{\mu}=\left\{X \mid \mathbb{E} V_{\mu}(X) \leq L_{\mu}\right\}=\left\{X \mid \mathbb{E}\left[\mu x^{2}+y^{2}+(z-\mu a-c)^{2}\right] \leq \frac{b^{2}(\mu a+c)^{2}}{4 \alpha(b-d)}\right\}, \mu \geq 0$

is globally exponential attractive set of system (1.2), where

$$
V_{\mu}(X)=\frac{1}{2}\left(\mu x^{2}+y^{2}+(z-\mu a-c)^{2}\right) .
$$

Proof Define the Lyapunov function on $\mathfrak{R}^{3}$

$$
V_{\mu}(X)=\frac{1}{2}\left(\mu x^{2}+y^{2}+(z-\mu a-c)^{2}\right) .
$$

Applying Itô's formula to (4.1), we have

$$
\begin{aligned}
d V_{\mu}(X)= & {\left[-\mu a x^{2}-d y^{2}-d z^{2}+b(\mu a+c) z+\frac{\mu \sigma_{1}^{2}}{2} x^{2}+\frac{\sigma_{2}^{2}}{2} y^{2}\right] d t } \\
& +\left(\frac{\mu \sigma_{1}}{2} x^{2}+\frac{\sigma_{2}}{2} y^{2}\right) d W(t)+\left[\mu\left(\gamma_{1}^{2}+2 \gamma_{1}\right) x^{2}+\left(\gamma_{2}^{2}+2 \gamma_{2}\right) y^{2}\right] d N(t) \\
= & {\left[-\left(\mu a-\frac{\mu \sigma_{1}^{2}}{2}\right) x^{2}-\left(d-\frac{\sigma_{2}^{2}}{2}\right) y^{2}-d z^{2}+2 d(\mu a+c) z-d(\mu a-c)^{2}\right] d t } \\
& +\left[(d-b) z^{2}+d(\mu a+c)^{2}+(b-2 d)(\mu a+c) z\right] d t \\
& +\left(\frac{\mu \sigma_{1}}{2} x^{2}+\frac{\sigma_{2}}{2} y^{2}\right) d W(t)+\left[\mu\left(\gamma_{1}^{2}+2 \gamma_{1}\right) x^{2}+\left(\gamma_{2}^{2}+2 \gamma_{2}\right) y^{2}\right] d N(t) \\
\leq & {\left[-\left(\mu a-\frac{\mu \sigma_{1}^{2}}{2}\right) x^{2}-\left(d-\frac{\sigma_{2}^{2}}{2}\right) y^{2}-d(z-\mu a-c)^{2}\right] d t } \\
& +\left[(d-b) z^{2}+d(\mu a+c)^{2}+(b-2 d)(\mu a+c) z\right] d t \\
& +\left(\frac{\mu \sigma_{1}}{2} x^{2}+\frac{\sigma_{2}}{2} y^{2}\right) d W(t)+\left[\mu\left(\gamma_{1}^{2}+2 \gamma_{1}\right) x^{2}+\left(\gamma_{2}^{2}+2 \gamma_{2}\right) y^{2}\right] d N(t) .
\end{aligned}
$$


Let

$$
f(z)=(d-b) z^{2}+(b-2 d)(\mu a+c) z,
$$

and

$$
f^{\prime}(z)=2(d-b) z+(b-2 d)(\mu a+c)=0,
$$

we have

$$
z_{0}=\frac{(b-2 d)(\mu a+c)}{2(b-d)},
$$

since $b>2 d$, then $z_{0}>0$, thus

$$
f^{\prime \prime}\left(z_{0}\right)=2(d-b)<0,
$$

therefore

$$
\sup _{z \in \Omega_{\mu}} f(z)=f\left(z_{0}\right)=\frac{(b-2 d)^{2}(\mu a+c)^{2}}{4(b-d)} .
$$

From (4.2) and (4.3), we can obtain

$$
\begin{aligned}
d V_{\mu}(X) \leq & {\left[-\left(\mu a-\frac{\mu \sigma_{1}^{2}}{2}\right) x^{2}-\left(d-\frac{\sigma_{2}^{2}}{2}\right) y^{2}-d(z-\mu a-c)^{2}\right.} \\
& \left.+d(\mu a+c)^{2}+\frac{(b-2 d)^{2}(\mu a+c)^{2}}{4(b-d)}\right] d t \\
& +\left(\frac{\mu \sigma_{1}}{2} x^{2}+\frac{\sigma_{2}}{2} y^{2}\right) d W(t)+\left[\mu\left(\gamma_{1}^{2}+2 \gamma_{1}\right) x^{2}+\left(\gamma_{2}^{2}+2 \gamma_{2}\right) y^{2}\right] d N(t) \\
= & \left.-\left(\mu a-\frac{\mu \sigma_{1}^{2}}{2}\right) x^{2}-\left(d-\frac{\sigma_{2}^{2}}{2}\right) y^{2}-d(z-\mu a-c)^{2}+\frac{b^{2}(\mu a+c)^{2}}{4(b-d)}\right] d t \\
& +\left(\frac{\mu \sigma_{1}}{2} x^{2}+\frac{\sigma_{2}}{2} y^{2}\right) d W(t)+\left[\mu\left(\gamma_{1}^{2}+2 \gamma_{1}\right) x^{2}+\left(\gamma_{2}^{2}+2 \gamma_{2}\right) y^{2}\right] d N(t) \\
\leq & {\left[-\left(d-\frac{\sigma_{2}^{2}}{2}\right)\left\{\mu x^{2}+y^{2}+(z-\mu a-c)^{2}\right\}+\frac{b^{2}(\mu a+c)^{2}}{4(b-d)}\right] d t } \\
& +\left(\frac{\mu \sigma_{1}}{2} x^{2}+\frac{\sigma_{2}}{2} y^{2}\right) d W(t)+\left[\mu\left(\gamma_{1}^{2}+2 \gamma_{1}\right) x^{2}+\left(\gamma_{2}^{2}+2 \gamma_{2}\right) y^{2}\right] d N(t) \\
\leq & {\left[-\left(d-\frac{\sigma_{2}^{2}}{2}\right) V_{\mu}(X)+\frac{b^{2}(\mu a+c)^{2}}{4(b-d)}\right] d t+\max \left\{\frac{\sigma_{1}}{2}, \frac{\sigma_{2}}{2}\right\} V_{\mu}(X) d W(t) } \\
& +\max \left\{\gamma_{1}^{2}+2 \gamma_{1}, \gamma_{2}^{2}+2 \gamma_{2}\right\} V_{\mu}(X) d N(t) .
\end{aligned}
$$


From (4.4) and the calculating the expectation we get

$$
\begin{aligned}
\mathbb{E} V_{\mu}(X) \leq & V_{\mu}\left(X_{0}\right)+\int_{t_{0}}^{t}\left[-\left(d-\frac{\sigma_{2}^{2}}{2}\right) \mathbb{E} V_{\mu}(X)+\frac{b^{2}(\mu a+c)^{2}}{4(b-d)}\right] d s \\
& +\int_{t_{0}}^{t} \lambda \max \left\{\gamma_{1}^{2}+2 \gamma_{1}, \gamma_{2}^{2}+2 \gamma_{2}\right\} \mathbb{E} V_{\mu}(X) d s .
\end{aligned}
$$

From (4.5), we can obtain

$$
\begin{aligned}
\mathbb{E} V_{\mu}(X) & \leq V_{\mu}\left(X_{0}\right) e^{-\alpha\left(t-t_{0}\right)}+K \int_{t_{0}}^{t} e^{-\alpha(t-s)} d s \\
& =V_{\mu}\left(X_{0}\right) e^{-\alpha\left(t-t_{0}\right)}+\frac{K}{\alpha}\left(1-e^{-\alpha\left(t-t_{0}\right)}\right)
\end{aligned}
$$

where

$\alpha=-\lambda \max \left\{\gamma_{1}^{2}+2 \gamma_{1}, \gamma_{2}^{2}+2 \gamma_{2}\right\}+\left(d-\frac{\sigma_{2}^{2}}{2}\right), \quad K=\frac{b^{2}(\mu a+c)^{2}}{4(b-d)}, L_{\mu}=\frac{K}{\alpha}$.

thus, when $\mathbb{E} V_{\mu}(X)-L_{\mu}>0, \mathbb{E} V_{\mu}\left(X_{0}\right)-L_{\mu}>0$, the following estimate holds on a globally exponential attractive set of system (1.2)

$$
\mathbb{E}\left[V_{\mu}(X)-L_{\mu}\right] \leq\left[V_{\mu}\left(X_{0}\right)-L_{\mu}\right] e^{-\alpha\left(t-t_{0}\right)} .
$$

By (4.7), we have

$$
\lim _{t \rightarrow \infty} \mathbb{E} V_{\mu}(X) \leq L_{\mu}
$$

that is

$\Omega_{\mu}=\left\{X \mid \mathbb{E} V_{\mu}(X) \leq L_{\mu}\right\}=\left\{X \mid \mathbb{E}\left[\mu x^{2}+y^{2}+(z-\mu a-c)^{2}\right] \leq \frac{b^{2}(\mu a+c)^{2}}{4 \alpha(b-d)}\right\}$

The proof of the theorem is complete.

Remark 4.1 If $\sigma_{1}=\sigma_{2}=\gamma_{1}=\gamma_{2}=0$ and $X_{0}$ deterministic then (1.2) implies that

$$
\Omega_{\mu}=\left\{X \mid V_{\mu}(X) \leq L_{\mu}\right\}=\left\{X \mid\left[\mu x^{2}+y^{2}+(z-\mu a-c)^{2}\right] \leq \frac{b^{2}(\mu a+c)^{2}}{4(b-d)}\right\} .
$$

We have prove for the deterministic case that there exists an attractor in to which every solution enters in finite time (If $\sigma_{1} \neq \sigma_{2} \neq \gamma_{1} \neq \gamma_{2} \neq 0$ then the limes superior of $\mathbb{E}\left[\mu x^{2}+y^{2}+(z-\mu a-c)^{2}\right]$ is bounded by $\left.\frac{b^{2}(\mu a+c)^{2}}{4 \alpha(b-d)}\right)$. 
Theorem 4 The stochastic Lorenz equation (1.2) generates a smooth random dynamical system $\varphi$ with jumps which is global to forward, that is $D_{t}=\Re^{3}$ for all $t>0$. Moreover, $\varphi\left(t, \theta_{-t} \omega, x\right)$ is $\mathcal{F}_{t}^{0}$-measurable

Proof Choose a function

$$
V(X)=\frac{1}{2}\left[y^{2}+(z-c)^{2}\right]
$$

From the proof of theorem 3 , let $\mu=0$ and Itô's formula yields

$$
\begin{aligned}
V(X) \leq & V\left(X_{0}\right)+\int_{0}^{t}\left[-\left(d-\frac{\sigma_{2}^{2}}{2}\right) V(X)+\frac{b^{2} c^{2}}{4(b-d)}\right] d s \\
& +\int_{0}^{t} \max \left\{\sigma_{1}, \sigma_{2}\right\} V(X) d W(s)+\int_{0}^{t} \max \left\{\gamma_{1}^{2}+2 \gamma_{1}, \gamma_{2}^{2}+2 \gamma_{2}\right\} V(X) d N(s) .
\end{aligned}
$$

Suppose that $Z$ satisfies the following equation with jumps

$$
\begin{aligned}
Z \leq & V\left(X_{0}\right)+\int_{0}^{t}\left[-\left(d-\frac{\sigma_{2}^{2}}{2}\right) Z_{s}+\frac{b^{2} c^{2}}{4(b-d)}\right] d s+\int_{0}^{t} \max \left\{\frac{\sigma_{1}}{2}, \frac{\sigma_{2}}{2}\right\} Z_{s} d W(s) \\
& +\int_{0}^{t} \max \left\{\gamma_{1}^{2}+2 \gamma_{1}, \gamma_{2}^{2}+2 \gamma_{2}\right\} Z_{s} d N(s) .
\end{aligned}
$$

Then $Z$ is finite on any finite interval $[0, T]$ for any $T>0$. Hence, (4.9) and (4.10) yield that the process $V(X)$ is finite on $[0, T]$ for any $\infty>T>0$, That is the random dynamical system $\varphi(t, \omega, x)$ is global to the forward. The proof of the theorem is complete.

Theorem 5 Let $a>0, b>2 d, c>0, d>0, a-d>\frac{\sigma_{1}^{2}-\sigma_{2}^{2}}{2}$,

$$
\left(d-\frac{\sigma_{2}^{2}}{2}\right)-\lambda \log \left(1+\max \left\{\gamma_{1}^{2}+2 \gamma_{1}, \gamma_{2}^{2}+2 \gamma_{2}\right\}\right)>0,
$$

then the random dynamical system generate by (1.2) possesses the unique parameter dependent tempered random attractor $A$ with domain of attraction $\mathcal{D}(A)$ containing the universe of sets $\mathcal{C l}(\mathcal{U})$, generated by

$$
\mathcal{U}=\left\{(D(\omega))_{\omega \in \Omega}: D(\omega) \subset \mathfrak{R}^{3} \text { is a tempered random set }\right\}
$$


Proof Define the Lyapunov function on $\mathfrak{R}^{3}$

$$
V_{\mu}(X)=\frac{1}{2}\left(x^{2}+y^{2}+(z-a-c)^{2}\right) .
$$

From the proof of theorem 3, let $\mu=1$ and Itô's formula yields

$$
\begin{aligned}
V(X) \leq & V\left(X_{0}\right)+\int_{0}^{t}\left[-\left(d-\frac{\sigma_{2}^{2}}{2}\right) V(X)+\frac{b^{2}(\mu a+c)^{2}}{4(b-d)}\right] d s \\
& +\int_{0}^{t} \max \left\{\frac{\sigma_{1}}{2}, \frac{\sigma_{2}}{2}\right\} V(X) d W(s)+\int_{0}^{t} \max \left\{\gamma_{1}^{2}+2 \gamma_{1}, \gamma_{2}^{2}+2 \gamma_{2}\right\} V(X) d N(s) .
\end{aligned}
$$

Denote $\varphi(t, \omega,(x, y, z))$ and $\psi(t, \omega, V(X))$ the random dynamical system which are generated by (1.2) and

$$
\begin{aligned}
Z_{t}= & \left.V\left(X_{0}\right)+\int_{0}^{t}\left[-\left(d-\frac{\sigma_{2}^{2}}{2}\right) Z_{s}\right)+\frac{b^{2}(\mu a+c)^{2}}{4(b-d)}\right] d s \\
& +\int_{0}^{t} \max \left\{\frac{\sigma_{1}}{2}, \frac{\sigma_{2}}{2}\right\} Z_{s} d W(s)+\int_{0}^{t} \max \left\{\gamma_{1}^{2}+2 \gamma_{1}, \gamma_{2}^{2}+2 \gamma_{2}\right\} Z_{s} d N(s) .
\end{aligned}
$$

respectively. The random dynamical system generated by by (4.13) is

$$
\begin{aligned}
& \psi(t, \omega) x=e^{-\left(d-\frac{\sigma_{2}^{2}}{2}\right) t+\max \left\{\frac{\sigma_{1}}{2}, \frac{\sigma_{2}}{2}\right\} W(t)+N_{t} \lg \left(1+\max \left\{\gamma_{1}^{2}+2 \gamma_{1}, \gamma_{2}^{2}+2 \gamma_{2}\right\}\right)} \\
& \times\left[V\left(X_{0}\right)+\frac{b^{2}(\mu a+c)^{2}}{4(b-d)} \int_{0}^{t} e^{\left(d-\frac{\sigma_{2}^{2}}{2}\right) s-\max \left\{\frac{\sigma_{1}}{2}, \frac{\sigma_{2}}{2}\right\} W(s)-N_{s} \lg \left(1+\max \left\{\gamma_{1}^{2}+2 \gamma_{1}, \gamma_{2}^{2}+2 \gamma_{2}\right\}\right)} d s\right] .
\end{aligned}
$$

From (4.12) yields

$$
V(\varphi(t, \omega,(x, y, z))) \leq \psi(t, \omega) V(X) .
$$

Thus $\psi(t, \omega)$ has the unique invariant measure which is a Dirac measure supported by

$\xi(\omega)=\frac{b^{2}(\mu a+c)^{2}}{4(b-d)} \int_{0}^{t} e^{\left(d-\frac{\sigma_{2}^{2}}{2}\right) s-\max \left\{\frac{\sigma_{1}}{2}, \frac{\sigma_{2}}{2}\right\} W_{s}-N_{s} \lg \left(1+\max \left\{\gamma_{1}^{2}+2 \gamma_{1}, \gamma_{2}^{2}+2 \gamma_{2}\right\}\right)} d s>0$. 
It is easy to prove that $\psi\left(t, \theta_{-t} \theta\right) x\left(\theta_{-t} \omega\right) \rightarrow \xi(\omega)$ as $t \rightarrow \infty$ for initial value $x(\omega) \in \Re_{+}$such that $e^{-\left(d-\frac{\sigma_{2}^{2}}{2}\right) t} x\left(\theta_{-t} \omega\right) \rightarrow 0$ for some $\left(d-\frac{\sigma_{2}^{2}}{2}\right)>0$. Hence, we may definite the universe of sets

$$
\overline{\mathcal{U}}=\left\{I(\omega) \subset \Re_{+} \text {is a tempered random set }\right\},
$$

that is, the random variable $\eta(\omega)=\sup _{x \in I(\omega)} x$ satisfies $\lim _{t \rightarrow \infty} \log ^{+}\left|\eta\left(\theta_{t} \omega\right) / t\right|=0$ for any $I \in \overline{\mathcal{U}}$. Then $\lim _{t \rightarrow \infty} e^{-\varepsilon t} \eta\left(\theta_{-t} \omega\right)=0$ for any $\varepsilon>0$.

It is obvious that $\overline{\mathcal{U}}$ is closed under inclusion and $\Omega \times\{x\} \in \overline{\mathcal{U}}$ for all $x \in \Re_{+}$.

Next. we will prove that the random set $[0,(1+\varepsilon) \xi]$ is forward invariant and absorbing for $\psi(t, \omega)$ with respect to the universe $\overline{\mathcal{U}}$ for any $\varepsilon>0$.

In fact, the absorbing property follows from the definition of $\overline{\mathcal{U}}$. We only prove that $\psi(t, \omega)[0,(1+\varepsilon) r(\omega)] \subset\left[0,(1+\varepsilon) r\left(\theta_{t} \omega\right)\right]$ for all $t \geq 0$. Since $\psi(t, \omega)$ is nonnegative function on $\Re$ and $\psi(t, \omega) x<\psi(t, \omega) y$ for $x<y$, it suffices to show $\psi(t, \omega)(1+\varepsilon) r(\omega) \leq(1+\varepsilon) r\left(\theta_{t} \omega\right)$, it is equivalent that prove

$$
\psi\left(t, \theta_{-t} \omega\right)(1+\varepsilon) r\left(\theta_{-t} \omega\right) \leq(1+\varepsilon) r(\omega)
$$

By (4.14), one has

$$
\begin{aligned}
\psi\left(t, \theta_{-t} \omega\right)(1+\varepsilon) r\left(\theta_{-t} \omega\right) & =r(\omega)+\varepsilon K \int_{-\infty}^{t} \exp \left(a s-2 \alpha_{1} B_{s}^{H_{1}}-2 \alpha_{2} B_{s}^{H_{2}}\right) d s \\
& \leq(1+\varepsilon) r(\omega)
\end{aligned}
$$

it implies that (4.17) is true.

Since

$$
\begin{aligned}
r\left(\theta_{-t} \omega\right) & =K \exp \left(a t-2 \alpha_{1} B_{-t}^{H_{1}}-2 \alpha_{2} B_{-t}^{H_{2}}\right) \int_{-\infty}^{-t} \exp \left(a s-2 \alpha_{1} B_{s}^{H_{1}}-2 \alpha_{2} B_{s}^{H_{2}}\right) d s \\
& \leq(r(\omega),
\end{aligned}
$$

one has

$$
e^{-\epsilon t} r\left(\theta_{-t} \omega\right) \rightarrow 0, \quad t \rightarrow \infty
$$

for any $\epsilon>0$.. It means that $[0,(1+\varepsilon) r(\omega)] \in \overline{\mathcal{U}}$ for any $\varepsilon>0$.

for any $\varepsilon>0$, define the subset $B(\omega)$ of $\Re^{2}$ by

$$
B(\omega)=V^{-1}([0,(1+\varepsilon)] r(\omega)) .
$$

This is a non-empty compact set by the surjectivity and continuity of $V(x, y)$, and the fact that pre-images of bounded sets are bounded under $V(x, y)$. 
Using the surjectivity and non-negativity of $V$ and (4.15), one has

$$
\begin{aligned}
\psi\left(t, \theta_{-t} \omega\right) & {\left[0,(1+\varepsilon) r\left(\theta_{-t} \omega\right)\right] \subset[0,(1+\varepsilon) r(\omega)] } \\
& \Leftrightarrow \psi\left(t, \theta_{-t} \omega\right) V\left(B\left(\theta_{-t} \omega\right)\right) \subset V(B(\omega)) \\
& \Rightarrow V\left(\psi\left(t, \theta_{-t} \omega\right) B\left(\theta_{-t} \omega\right)\right) \subset V(B(\omega))
\end{aligned}
$$

for all $t \geq 0$. It implies that

$$
\psi\left(t, \theta_{-t} \omega\right) B\left(\theta_{-t} \omega\right) \subset V^{-1}\left(V\left(\psi\left(t, \theta_{-t} \omega\right) B\left(\theta_{-t} \omega\right)\right)\right) \subset V^{-1}(V(B(\omega))=B(\omega),
$$

that is, $B$ is forward invariant.

As the proof of (4.19) we have that there is a $t(\omega, D)>0$ such that, for all $t>t(\omega, D)$,

$$
V\left(\varphi\left(t, \theta_{-t} \omega\right) D\left(\theta_{-t} \omega\right)\right) \subset \psi\left(t, \theta_{-t} \omega\right) V\left(D\left(\theta_{-t} \omega\right)\right) \subset[0,(1+\varepsilon) r(\omega)]=V(B(\omega))
$$

by $(4.17)$ and the set $[0,(1+\varepsilon) r(\omega)]$ absorbing any set in $\overline{\mathcal{U}}$. It shows the absorption of any set in $\mathcal{U}$.

Finally, we prove the existence of a neighborhood of $B$ in $\mathcal{U}$. In fact, for any $B \in \mathcal{U}$, Proposition 3.2(iii) of Schenk-Hoppé [11] implies that there exist a random variable $\rho(\omega)>0$ such that $B(\omega) \subset S(\rho(\omega))=\left\{x \in \mathfrak{R}^{2},\|x\| \leq \rho(\omega)\right\}$. It is clear that $S(b \rho(\omega)) \in \mathcal{U}$ and that $S(\rho(\omega))$ is a neighborhood of $B$ for all $b>1$. The proof of the theorem is complete.

\section{Stochastic bifurcation}

In this section, we will analyze the bifurcation behavior of the randomly perturbed Hopf bifurcation of the stochastic Lorenz family of chaotic systems with jump, using the theory of random attractors.

Proposition 1 Suppose $a>\frac{\sigma_{1}^{2}}{2}, d>\frac{\sigma_{2}^{2}}{2}$, we have that the top Lyapunov exponents $l$ of the linear stochastic equation

$$
\left\{\begin{array}{l}
\dot{x}(t)=a(y-x)+\sigma_{1} x d W(t)+\gamma_{1} y d N(t) \\
\dot{y}(t)=c x-d y+\sigma_{2} y d W(t)+\gamma_{2} x d N(t) \\
\dot{z}(t)=-b z
\end{array}\right.
$$

is bounded by

$l \leq-\min \left\{\left(a-\frac{\sigma_{1}^{2}}{2}\right),\left(d-\frac{\sigma_{2}^{2}}{2}\right), b\right\}+\lambda \log \left[1+\max \left\{\left(2 \gamma_{1}+\gamma_{1}^{2}\right),\left(2 \gamma_{2}+\gamma_{2}^{2}\right)\right\}\right]$.

Proof Define the Lyapunov function on $\mathfrak{R}^{3}$

$$
V(X)=\frac{1}{2}\left(c x^{2}+a y^{2}+z^{2}\right)
$$


yields $V(X) \geq 0$ and $V(X)=0$ if and only $x=y=z=0$. Itô's formula implies

$$
\begin{aligned}
d V(X)= & {\left[c a x(y-x)+a y(c x-d y)-b z^{2}+\frac{c \sigma_{1}^{2}}{2} x^{2}+\frac{a \sigma_{2}^{2}}{2} y^{2}\right] d t } \\
& +\left[c \sigma_{1} x^{2}+a \sigma_{2} y^{2}\right] d W(t)+\left[c\left(2 \gamma_{1}+\gamma_{1}^{2}\right) x^{2}+a\left(2 \gamma_{2}+\gamma_{2}^{2}\right) y^{2}\right] d N(t) \\
= & {\left[-c\left(a-\frac{\sigma_{1}^{2}}{2}\right) x^{2}-a\left(d-\frac{\sigma_{2}^{2}}{2}\right) y^{2}-b z^{2}\right] d t } \\
& +\left[\frac{c \sigma_{1}}{2} x^{2}+\frac{a \sigma_{2}}{2} y^{2}\right] d W(t)+\left[c\left(2 \gamma_{1}+\gamma_{1}^{2}\right) x^{2}+a\left(2 \gamma_{2}+\gamma_{2}^{2}\right) y^{2}\right] d N(t) \\
\leq & \min \left\{\left(a-\frac{\sigma_{1}^{2}}{2}\right),\left(d-\frac{\sigma_{2}^{2}}{2}\right), b\right\} V(X) d t \\
& +\max \left\{\frac{\sigma_{1}}{2}, \frac{\sigma_{2}}{2}\right\} V(X) d W(t)+\max \left\{\left(2 \gamma_{1}+\gamma_{1}^{2}\right),\left(2 \gamma_{2}+\gamma_{2}^{2}\right)\right\} V(X) d N(t) .
\end{aligned}
$$

From (5.2) and Doléans-Dade's exponent formula, we can obtain

$$
V(X) \leq \exp \left\{-\rho t+\max \left\{\frac{\sigma_{1}}{2}, \frac{\sigma_{2}}{2}\right\}+N_{t} \log \left(1+\max \left\{\left(2 \gamma_{1}+\gamma_{1}^{2}\right),\left(2 \gamma_{2}+\gamma_{2}^{2}\right)\right\}\right)\right\}
$$

where

$$
\rho=\min \left\{\left(a-\frac{\sigma_{1}^{2}}{2}\right),\left(d-\frac{\sigma_{2}^{2}}{2}\right), b\right\} .
$$

We then have

$$
\begin{aligned}
l & =\lim _{t \rightarrow \infty} \frac{\log V(X)}{t} \\
& \leq \lim _{t \rightarrow \infty} \frac{-\rho t+\max \left\{\frac{\sigma_{1}}{2}, \frac{\sigma_{2}}{2}\right\} W(t)+N_{t} \log \left[1+\max \left\{\left(2 \gamma_{1}+\gamma_{1}^{2}\right),\left(2 \gamma_{2}+\gamma_{2}^{2}\right)\right\}\right]}{t} \\
& =-\rho+\lambda \log \left[1+\max \left\{\left(2 \gamma_{1}+\gamma_{1}^{2}\right),\left(2 \gamma_{2}+\gamma_{2}^{2}\right)\right\}\right] .
\end{aligned}
$$

The proof of the theorem is complete.

Theorem 6 suppose $a>2 \sigma_{1}^{2}, \mu d>2 \sigma_{2}^{2}$ and $\mu>0$ such that

$$
-\min \left\{\left(a-\frac{\sigma_{1}^{2}}{2}\right),\left(\mu d-\frac{\sigma_{2}^{2}}{2}\right), \mu b\right\}+\lambda \log \left[1+\max \left\{\left(2 \gamma_{1}+\gamma_{1}^{2}\right),\left(2 \gamma_{2}+\gamma_{2}^{2}\right)\right\}\right]<0
$$


Then, the random dynamical system generate by the stochastic Duffing van der Pol equation with $\mathrm{fBm}(3.1)$ possesses the unique tempered random attractors $A(\omega)=\{0\}$ with domain of attraction $\mathcal{D}(A)$ containing the universe of $\mathrm{Cl}(\mathcal{U})$ given by

$$
\mathcal{U}=\left\{\left(D(\omega)_{\omega \in \Omega}\right): D(\omega) \subset \mathfrak{R}^{3} \text { is a tempered random set }\right\}
$$

where $C l(\mathcal{U})$ is the completion universe of $\mathcal{U}$ under inclusion.

In particular, for any $x \in \mathfrak{R}^{2}$, the solutions $\varphi\left(t, \theta_{-t} \omega\right) x$ and $\varphi(t, \omega) x$ tend to zero exponentially fast as $t \rightarrow \infty$.

Proof Proposition 4.2 and the assumption (5.4) yield that the random dynamical system generated (1.2) is stability. Define the Lyapunov function on $\mathfrak{R}^{3}$

$$
V(X)=\frac{1}{2}\left(x^{2}+\mu y^{2}+\mu\left(z-\frac{a+\mu c}{\mu}\right)^{2}\right)
$$

yields $V(X) \geq 0$ and $V(X)=0$ if and only $x=y=z=0$. Itô's formula implies

$$
\begin{aligned}
d V(X)= & {\left[-\left(a-\frac{\sigma_{1}^{2}}{2}\right) x^{2}-\left(\mu d-\frac{\sigma_{2}^{2}}{2}\right) y^{2}-\mu b z^{2}+\frac{b(a+\mu c)}{\mu} z\right] d t } \\
& -\left(\frac{\sigma_{1}}{2} x^{2}+\frac{\mu \sigma_{2}}{2} y^{2}\right) d W(t)+\left[\left(2 \gamma_{1}+\gamma_{1}^{2}\right) x^{2}+\mu\left(2 \gamma_{2}+\gamma_{2}^{2}\right) y^{2}\right] d N(t) \\
\leq & {\left[-\left(a-\frac{\sigma_{1}^{2}}{2}\right) x^{2}-\left(\mu d-\frac{\sigma_{2}^{2}}{2}\right) y^{2}-\mu b\left(z-\frac{a+\mu c}{\mu}\right)^{2}+\frac{b(a+\mu c)^{2}}{2 \mu}\right] d t } \\
& +\max \left\{\frac{\sigma_{1}}{2}, \frac{\sigma_{2}}{2}\right\} V(X) d W(t)+\max \left\{\left(2 \gamma_{1}+\gamma_{1}^{2}\right),\left(2 \gamma_{2}+\gamma_{2}^{2}\right)\right\} V(X) d N(t) \\
\leq & {\left[-\Lambda V(X)+\frac{b(a+\mu c)^{2}}{2 \mu}\right] d t+\max \left\{\frac{\sigma_{1}}{2}, \frac{\sigma_{2}}{2}\right\} V(X) d W(t) } \\
& +\max \left\{\left(2 \gamma_{1}+\gamma_{1}^{2}\right),\left(2 \gamma_{2}+\gamma_{2}^{2}\right)\right\} V(X) d N(t),
\end{aligned}
$$

where

$$
\Lambda=\min \left\{\left(a-\frac{\sigma_{1}^{2}}{2}\right),\left(\mu d-\frac{\sigma_{2}^{2}}{2}\right), \mu b\right\} .
$$

Corollary 5.63. of Arnold [22] yields that the random dynamical system generated by the stochastic differential equation with jump

$$
\begin{aligned}
d Z_{t}= & {\left[-\Lambda Z_{T}+\frac{b(a+\mu c)^{2}}{2 \mu}\right] d t+\max \left\{\frac{\sigma_{1}}{2}, \frac{\sigma_{2}}{2}\right\} Z_{T} d W(t) } \\
& +\max \left\{\left(2 \gamma_{1}+\gamma_{1}^{2}\right),\left(2 \gamma_{2}+\gamma_{2}^{2}\right)\right\} Z_{t} d N(t)
\end{aligned}
$$


has the unique invariant measure $\delta_{0}$ which is the Dirac measure at 0 . As the proof in Theorem 5, one has that $A(\omega)=\{0\}=V^{-1}(\{0\})$ is the unique random attractor attracting any set form $\mathcal{U}$.

Hence, it means that that, for any $X \in \mathfrak{R}^{3}, \varphi\left(t, \theta_{-t} \omega\right) x$ and $\varphi(t, \omega) x$ tend to zero as $t \rightarrow \infty$ with an exponential fast. The proof of the theorem is complete.

\section{Numerical simulation results}

According to our analytical results the stochastic Lorenz family of chaotic systems are both exponentially stable and tend to zero under conditions specified in Theorems 1 and 2. We now try and support our analytical results by simulations (Figs. 1, 2, 3).

For the Lorenz family of chaotic systems, Formula (8) given in Theorem 3 can provide estimations for the boundaries of the attractive sets. The numerical results indicate that except for the case $\sigma_{i}$, all other cases exhibit either generalized Lorenz attractor or generalized Chen attractor. To illustrate the stochastic effects clearly we performed simulations first for the deterministic case (Fig. 4) and then for a corresponding stochastic simulation (Figs. 5, 6, 7). The parameter values used have all been taken from published [5,7]. The typical values for $a, b, c$ and $d$ are chosen as $a=10, b=8 / 3, c=28$ and $d=1$, and the values of $\sigma_{i}$ are varied from 0 to 3 .

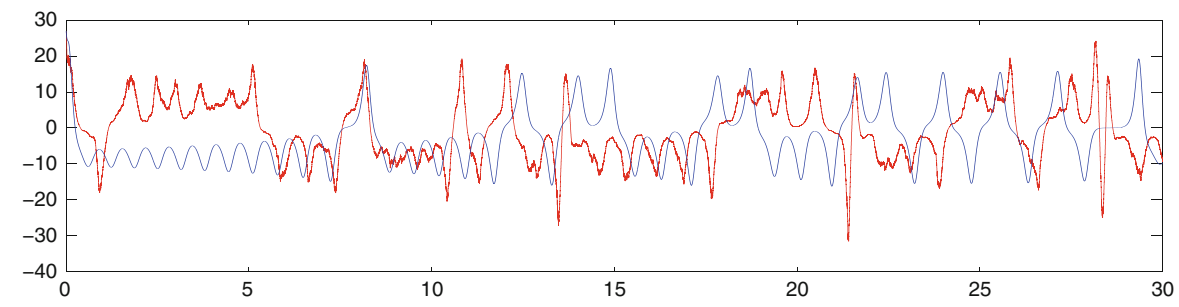

Fig. 1 Simulated phase portraits of the stochastic Lorenz family of chaotic systems (1.2) with the initial conditions $a=10, b=8 / 3, c=28$ and $d=1, \sigma_{1}=\sigma_{2}=0.5, \gamma_{1}=\gamma_{2}=0.01, \lambda=0.1$. Blue represent the simulation of the $x$ of determine Lorenz family of chaotic systems. Red represent the simulation of the $x$ of stochastic Lorenz family of chaotic systems (Color figure online)

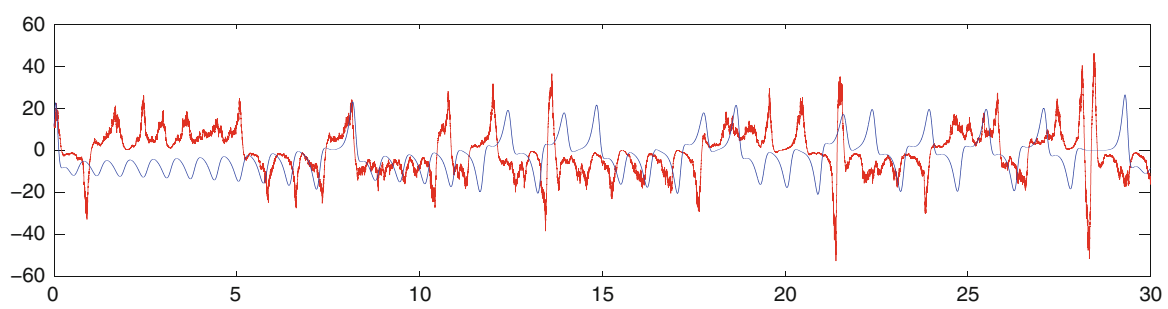

Fig. 2 Simulated phase portraits of the stochastic Lorenz family of chaotic systems (1.2) with the initial conditions $a=10, b=8 / 3, c=28$ and $d=1, \sigma_{1}=\sigma_{2}=0.5, \gamma_{1}=\gamma_{2}=0.01, \lambda=0.1$. Blue represent the simulation of the $y$ of determine Lorenz family of chaotic systems. Red represent the simulation of the $y$ of stochastic Lorenz family of chaotic systems (Color figure online) 


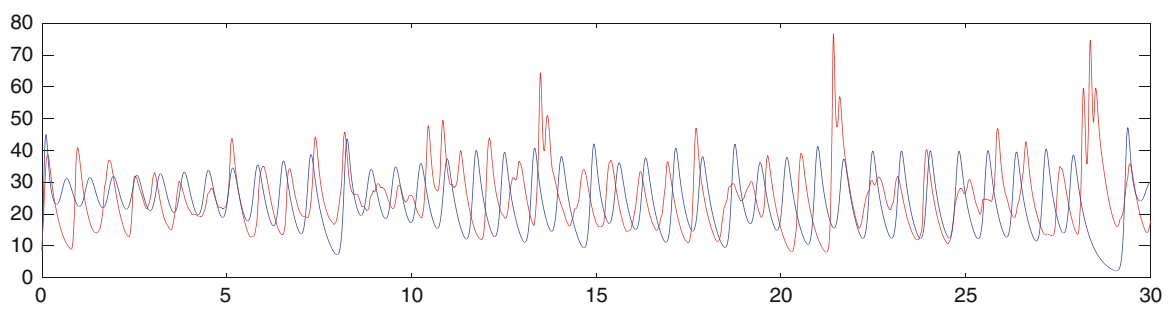

Fig. 3 Simulated phase portraits of the stochastic Lorenz family of chaotic systems (1.2) with the initial conditions $a=10, b=8 / 3, c=28$ and $d=1, \sigma_{1}=\sigma_{2}=0.5, \gamma_{1}=\gamma_{2}=0.01, \lambda=0.1$. Blue represent the simulation of the $z$ of determine Lorenz family of chaotic systems. Red represent the simulation of the $z$ of stochastic Lorenz family of chaotic systems (Color figure online)

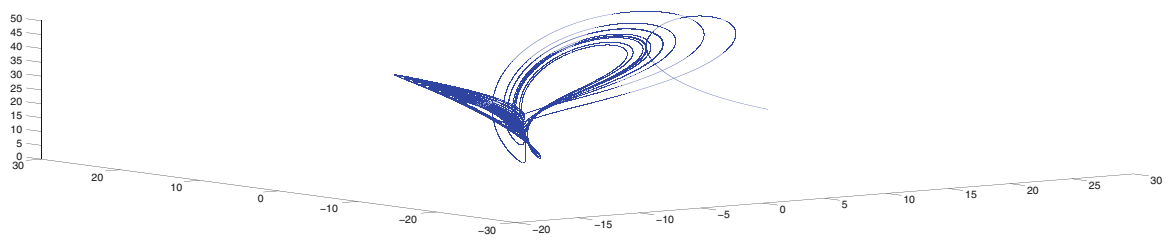

Fig. 4 The determine Lorenz family of chaotic systems (1.2) with the initial conditions $a=10, b=$ $8 / 3, c=28$ and $d=1, \sigma_{1}=\sigma_{2}=\gamma_{1}=\gamma_{2}=\lambda=0$

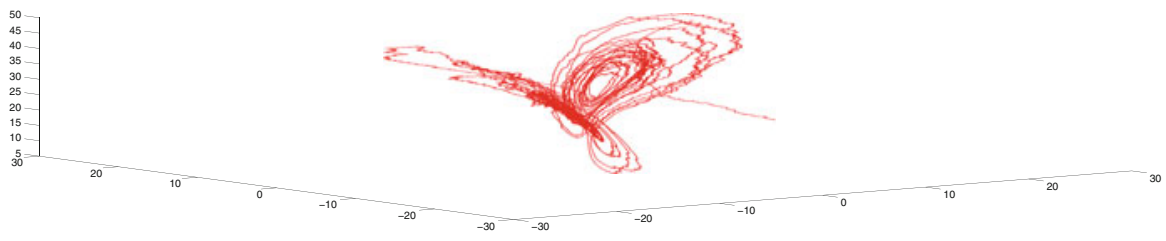

Fig. 5 Simulated phase portraits of the stochastic Lorenz family of chaotic systems (1.2) with the initial conditions $a=10, b=8 / 3, c=28$ and $d=1, \sigma_{1}=\sigma_{2}=0.5, \gamma_{1}=\gamma_{2}=0.01, \lambda=0.1$

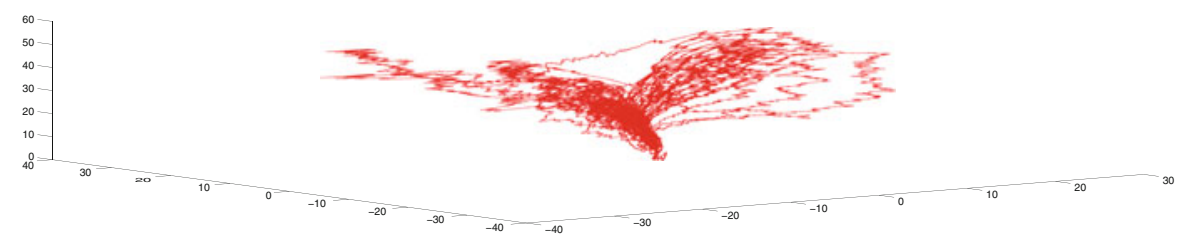

Fig. 6 Simulated phase portraits of the stochastic Lorenz family of chaotic systems (1.2) with the initial conditions $a=10, b=8 / 3, c=28$ and $d=1, \sigma_{1}=\sigma_{2}=2, \gamma_{1}=\gamma_{2}=0.01, \lambda=0.1$

Acknowledgments This research was supported by the National Natural Science Foundation of China (No. 11201089) and and (No. 11301090). Guangxi Natural Science Foundation (No. 2013GXNSFAA019014) and (No. 2013GXNSFBA019016). 


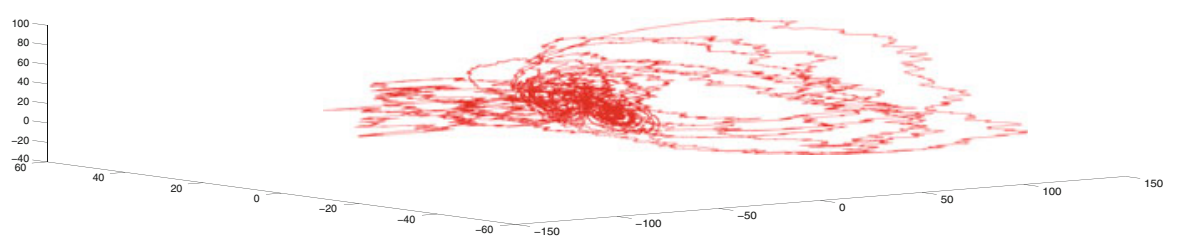

Fig. 7 Simulated phase portraits of the stochastic Lorenz family of chaotic systems (1.2) with the initial conditions $a=10, b=8 / 3, c=28$ and $d=1, \sigma_{1}=1, \sigma_{2}=3, \gamma_{1}=\gamma_{2}=0.01, \lambda=0.1$

Open Access This article is distributed under the terms of the Creative Commons Attribution License which permits any use, distribution, and reproduction in any medium, provided the original author(s) and the source are credited.

\section{References}

1. E. Lorenz, Deterministic nonperiodic flow. J. Atmospheric Sci. 20, 130-141 (1963)

2. G. Chen, T. Ueta, Yet another chaotic attractor. Int. J. Bifurcation Chaos 9, 1465-1466 (1999)

3. J. Lü, G. Chen, Anew chaotic attractor coined. Int. J. Bifurcation Chaos 12, 659-6661 (2002)

4. T. Zhou, G. Chen, Y. Tang, Auniversal unfolding of the Lorenz system. Chaos Solitons Fractals 20, 979-993 (2004)

5. X. Chen, Lorenz equations part I: existence and nonexistence of homoclinic orbits. SIAM J. Math. Anal. 27, 1057-1069 (1995)

6. A. Pogromsky, G. Santoboni, H. Nijmeijer, An ultimate bound on the trajectories of the Lorenz systems and its applications. Nonlinearity 16, 1597-1605 (2003)

7. C. Sparrow, The Lorenz Equations: Bifurcation, Chaos, and Strange Attractor (Springer, Berlin, 1982)

8. I. Stewart, The Lorenz attractor exists. Nature 406, 948-949 (2002)

9. S. Čelikovský, G. Chen, On a generalized Lorenz canonical form of chaotic systems. Int. J. Bifurcation Chaos 12, 1789-1812 (2002)

10. P. Yu, X. Liao, New estimates for globally attracvtive and positive invariant set ofyhe family of the Lorenz system. Int J Bifurcation Chaos 16, 3383-3390 (2006)

11. W. Tucker, The Lorenz attractor exists. C. R. Acad. Sci. Paris 328, 1197-1202 (1999)

12. G. Leonov, Lyapunov's direct method in the estimation of the Hausdorff dimension of attractors. Acta Appl. Math. 26, 1-60 (1992)

13. G. Leonov, Bound for attractors and the existence of homoclinic orbits in the Lorenz system. J. Appl. Math. 65, 19-32 (2001)

14. X. Liao, On the new results of global attractive set and positive invariant set of the Lorenz chaotic system and the application to chaos control and synchronization. Sci. China 34, 1-16 (2004)

15. X. Liao, Y. Fu, S. Xie et al., Globally exponentially attractive sets of family of Lorenz systems. Sci. China Ser. F-Inf. Sci. 51, 283-292 (2008)

16. D. Li, J. Lu, Estimating the bounds for the Lorenz family of chaotic systems. Chaos Solitons Fractals 23, 529-534 (2005)

17. L. Arnold, Stochastic Differential Equations: Theory and Applications (Wiley, New York, 1972)

18. H. Keller, Attractors and bifurcation of the stochastic Lorenz system, Technical Report 389, institut für Dynamische syteme, Universität Bremen 1996

19. B. Schmalfuß, The random attractor of stochastic Lorenz system. Z. Angew Math. Phys. 48, 951-975 (1997)

20. X. Liao, Q. Luo, Algebraic necessary and sufficient conditions for Lyapunov stability of Lorenz chaotic system and its application. Sci. China Ser. Inf. Sci. 8, 1086-1095 (2010)

21. Q. Yang, G. Chen, T. Zhou, A unified Lorenz-type system and its canonical form. Int. J. Bifurcation Chaos 16, 2855-2871 (2006)

22. L. Arnold, Random Dynamical Systems (Springer, New York, 1998) 
23. W. Zhu, Nonlinear Stochastic Dynamics and Control in Hamiltonian Formulation (Science Press, Beijing, 2003)

24. K. Schenk-Hoppé, Stochastic hopf bifurcation: an example. Inc. J. Non-Ltnmr Mechanws 31, 685-692 (1996)

25. Z. Huang, Q. Yang, J. Cao, Stochastic stability and bifurcation for the chronic state in marchuk's model with noise. Appl. Math. Model. 35, 5842-5855 (2011)

26. Z. Huang, Q. Yang, J. Cao, Complex dynamics in a stochastic internal HIV model. Chaos Solitons Fractals 54, 954-963 (2011)

27. N. Namachchivaya, Stochastic bifurcation. Appl. Math. Comput. 38, 101-159 (1990)

28. D. Huang, H. Wang, J. Feng, Z. Zhu, Hopf bifurcation of the stochastic model on hab nonlinear stochastic dynamics. Chaos Solitons Fractals 27, 1072-1079 (2006)

29. X. Zou, D. Fan, K. Wang, Stationary distribution and stochastic Hopf bifurcation for a predator-prey system with noise. Discret. Contin. Dyn. Syst. 18, 1507-1519 (2013)

30. Y. Xie, The random attractors of stochastic Duffing-van der Pol equations with jumps. Chin. J. Appl. Probab. Stat. 26, 9-23 (2010)

31. K. Schenk-Hoppé, Random attractors general properties, existence and application to stochastic bifurcation theory. Discret. Contin. Dyn. Syst. 4, 99-130 (1998) 